\title{
RESISTÊNCIA DO TIPO ANTIBIOSE A NINFAS DE TIBRACA LIMBATIVENTRIS (STAL, 1860) (HETEROPTERA: PENTATOMIDAE) EM VARIEDADES DE ARROZ
}

\author{
J.R. de Souza ${ }^{1 *}$, E. Ferreira ${ }^{2 \text { }}$, E.F. das Chagas ${ }^{3}$, J.M. Mondego ${ }^{3}$, \\ A.A.S. Maciel ${ }^{3}$, D.H.S. Sardinha ${ }^{3}$, K.K.M. Gonçalves ${ }^{3}$, J.A. Carvalho Filho ${ }^{3}$
}

${ }^{1}$ Universidade Estadual Paulista, Faculdade de Ciências Agrárias e Veterinárias, Departamento de Ciências Exatas, Via de Acesso Prof. Paulo Donato Castellane s/no, CEP 14849-900, Jaboticabal, SP, Brasil. E-mail: joseaneagro@yahoo.com.br

\section{RESUMO}

O percevejo-do-colmo (Tibraca limbativentris Stal, 1860) é uma importante praga do arroz no Brasil. O uso de variedades resistentes a esse inseto é uma ferramenta importante para o seu controle. O experimento foi conduzido em casa de vegetação na Embrapa Arroz e Feijão. Foi avaliada resistência tipo antibiose em ninfas de T. limbativentris em 16 variedades de arroz em delineamento experimental de blocos casualizados em oito repetições. Os caracteres utilizados para detectar indícios de resistência do tipo antibiose foram: número de ninfas vivas, massa seca (mg)/ninfas, superfície corporal $/ \mathrm{mm}^{2}$, índice de sobrevivência e desenvolvimento e dias de vida das ninfas. Realizaram-se três tipos de análises: a) considerando somente os dados obtidos nas repetições avaliadas aos 7, 13, 21 e 26 dias após a infestação; b) considerando somente os dados das quatro repetições avaliadas aos 35 dias após a infestação e c) considerando os dados obtidos conjuntamente nas oito repetições. Conclui-se que as variedades Arroz Comum e Desconhecido Branco, principalmente a primeira, evidenciam possuir características que lhes confere determinado grau de resistência do tipo antibiose a ninfas de T. limbativentris.

PALAVRAS-CHAVE: Oryza sativa, resistência de plantas a insetos, percevejo do colmo.

\section{ABSTRACT}

ANTIBIOSIS RESISTANCE TO THE NYMPHS OF TIBRACA LIMBATIVENTRIS (STAL, 1860) (HETEROPTERA: PENTATOMIDAE) IN VARIETIES OF RICE. The stem bug (Tibraca limbativentris Stal, 1860) is one of the most harmful rice pests in Brazil. Resistant varieties can be an important method for controlling this pest. The present experiment was carried out in the greenhouse at Embrapa Arroz e Feijão. Evaluation was made of the antibiosis to nymphs of T. limbativentris in 16 rice varieties in an experimental design of randomized blocks in 8 replications. The parameters evaluated were the number of live nymphs, dry matter-mg, body surface-mm2, nymphs survival and development index, and insect days of life. There were three types of tests: a) considering only the data obtained in replications evaluated at 7, 13, 21 and 26 days after the infestation; b) considering only the data from 4 replications evaluated at 35 days after the infestation and; c) considering the data together on 8 replications. It was concluded that the varieties Arroz Comum and Desconhecido Branco, especially the former, showed some degree of antibiosis resistance to nymphs of T. limbativentris.

KEY WORDS: Oryza sativa, host-plant insect resistance, stem bug.

\section{INTRODUÇÃO}

O ataque de insetos-praga é um dos principais fatores que reduzem a produtividade da cultura do arroz no Brasil. O percevejo-do-colmo Tibraca limbativentris (Stal, 1860) destaca-se como um dos insetos mais prejudiciais à cultura, sendo abundante na maioria das regiões orizícolas brasileiras. Em al-

${ }^{2}$ Embrapa Arroz e Feijão/CNPAF, Santo Antônio de Goiás, GO, Brasil.

${ }^{3}$ Universidade Estadual do Maranhão, Departamento de Fitotecnia e Fitossanidade, São Luiz, MA, Brasil.

*Mestranda em Agronomia - Genética e Melhoramento de Plantas, Universidade Estadual Paulista, Faculdade de Ciências Agrárias e Veterinárias.

"In memorian. 
guns anos, dependendo da abundância, pode causar prejuízos de até $90 \%$ na produtividade de grãos. Devido ao hábito de estabelecer-se e manter-se, predominantemente, entre os colmos, à base das plantas, nem sempre as infestações são percebidas na época mais recomendável para a adoção de medidas de controle (FERREIRA et al., 1997).

O percevejo ataca plantas com mais de 20 dias de idade, sendo os danos caracterizados por uma necrose parcial ou total da parte central dos colmos, em decorrência da injeção de saliva tóxica. A perfuração dos colmos na base das plantas, na fase vegetativa, provoca o sintoma de coração morto. $\mathrm{Na}$ fase reprodutiva, a perfuração na parte superior dos colmos origina $o$ sintoma de panícula branca, ou panículas com elevada porcentagem de espiguetas vazias. Mesmo que a necrose não se expanda ao restante dos tecidos das plantas atacadas, ocorre um retardamento de crescimento (FERREIRA et al., 1997).

Antecedentes de pesquisa indicam que o percevejodo-colmo, mesmo em populações relativamente baixas, causa severos danos à cultura do arroz. Estudos demonstraram que os danos são diferenciados, dependendo da fase fenológica da planta em que ocorre a infestação (CosTA; LINK, 1992), podendo cada percevejo, na fase reprodutiva, provocar a perda média de 3,2 colmos. Um percevejo reduz a produtividade em cerca de $59 \mathrm{~kg} / \mathrm{ha}$ e $65 \mathrm{~kg} / \mathrm{ha}$, se atacar as plantas de arroz, na fase vegetativa e reprodutiva, respectivamente. Uma infestação de um percevejo adulto $/ \mathrm{m}^{2}$ de arrozal é suficiente para que ocorra dano econômico (FERreIra et al., 1997).

Dentre os métodos de controle disponíveis para o percevejo-do-colmo, predomina a aplicação de inseticidas químicos (Ferreira; Martins, 1984). A utilização dos inseticidas químicos tem causado nos últimos anos um desequilíbrio nos ecossistemas por poluírem o meio ambiente, atuarem sobre os inimigos naturais dos insetos-pragas e promoverem o surgimento de populações de insetos-pragas resistentes. Além dessas conseqüências, este tipo de controle, muitas vezes, não possui eficácia desejada e onera os custos de produção (Tremiliosi, 2006). Portanto, perante a necessidade de alternativas ao controle químico, aliada à crescente demanda da sociedade por métodos menos agressivos ao meio ambiente, justifica-se a busca por outros métodos de controle do inseto.

Entre tais métodos, o emprego de variedades resistentes é considerado ideal, uma vez que as populações das pragas podem ser mantidas abaixo de seus níveis de danos, reduzindo distúrbios ambientais e sem exigir conhecimentos específicos do agricultor, nem custos adicionais. Dentre os tipos de resistência a antibiose caracteriza-se por proporcionar a redução da população de pragas, exercendo um efeito adverso ou mesmo letal sobre a biologia do inseto (LARA,1991).
Há pouca informação sobre a resistência de variedades de arroz à T. limbativentris, a pesar da realização de alguns trabalhos a respeito (TRUjILlo, 1970; FERreira et al., 1986; SOUSA; RODRIGUES, 1990). Ainda não foram desenvolvidas nem selecionadas variedades de arroz resistentes ao inseto utilizáveis comercialmente, sendo que a seleção de variedades com essa característica seria baseada, principalmente, em alta capacidade de afilhamento e associada a um menor número de afilhos danificados (FERREIRA et al., 1997).

Inúmeras pesquisas abordam o uso de variedades resistentes a insetos-praga como um dos componentes de programas de manejo ecológico em várias culturas (Ferreira; Lara, 1999; Viana; Potenza, 2000; SuINAGA et al., 2004). Essas pesquisas podem servir de base a estudos sobre resistência de arroz à $T$. limbativentris, como este trabalho cujo objetivo é identificar variedades com resistência do tipo antibiose a ninfas, visando a utilização efetiva como componente de sistemas de manejo integrado do inseto.

\section{MATERIAL E MÉTODOS}

O experimento foi conduzido no período de fevereiro a abril de2005, em casa de vegetação, na Embrapa Arroz e Feijão, no Município de Santo Antônio de Goiás, GO. Foram utilizadas 15 variedades de arroz consideradas tradicionais e cultivadas no Estado do Maranhão, provenientes de estudos conduzidos por SouzA et al. (2006) e uma testemunha a variedade BR IRGA 409, considerada suscetível ao T.limbativentris.

O delineamento experimental foi o de blocos casualizados com dezesseis tratamentos em oito repetições. As 16 variedades foram semeadas (17/2/ 2005) em covas com densidade de 15 sementes espaçadas em 0,40 m. Vinte dias após a semeadura as plantas de cada tratamento foram mantidas em gaiolas cilíndricas com base de PVC $(0,25 \mathrm{~m}$ de diâmetro x 0,20 m de altura) revestida por tecido fino ("voile"). Cinco dias após, as plantas foram contadas e infestadas com 10 ninfas de $2^{\circ}$ instar/cova com idade máxima de 5 horas após a primeira ecdise, provenientes de adultos alimentados em plantas da cultivar de arroz irrigado BR IRGA 409. Quando surgiram insetos adultos na variedade testemunha, cerca de 35 dias após a infestação, o experimento foi encerrado. Os insetos foram coletados, acondicionados em sacos de papel e mantidos em congelador por 24 horas. Posteriormente, foram secos em estufa a $50^{\circ} \mathrm{C}$ por 36 horas e pesados em balança de precisão.

Quatro repetições foram individualmente avaliadas aos 7, 13, 21 e 26 dias, as demais 35 dias após a infestação. Os caracteres utilizados para detectar indícios de resistência do tipo antibiose foram: número deninfas vivas (NNV), massa seca (mg)/ninfa (MSN), 
superfície corporal $/ \mathrm{mm}^{2}$ (SPN), índice de sobrevivência e desenvolvimento (ISD) e dias de vida das ninfas (DDV). Os dias de vida das ninfas nas parcelas foram obtidos multiplicando-se o número de ninfas vivas pelo número de dias decorridos até o encerramento das avaliações em cada repetição. $O$ índice de sobrevivência e desenvolvimento consistiu em setransformar o número de ninfas de diferentes tamanhos em número equivalente de adultos, fazendo-se um adulto igual a quatro ninfas de primeiro instar, ou duas ninfas de segundo instar ou 0,75 ninfas de terceiro instar (FERREIRA, comunicação pessoal). A massa seca/ ninfa resultou da divisão da massa seca total de cada parcela pelo número de insetos encontrados.

As variáveis foram analisadas sem e com transformação em $\sqrt{X+0,5}$. Transformaram-se o número de ninfas vivas, massa seca $(\mathrm{mg})$, superfície corporal $\left(\mathrm{mm}^{2}\right)$ e os dias de vida das ninfas. Utilizou-se o programa SAS (1990), sendoas médias comparadas pelo Testede Duncan ao nível de $5 \%$ de probabilidade. Realizaramse três tipos de análises: a) considerando somente os dados obtidos nas repetições avaliadas aos 7, 13, 21 e 26 dias após a infestação; b) considerando somente os dados das quatro repetições avaliadas aos 35 dias após a infestação e c) considerando os dados obtidos conjuntamente nas oito repetições.

\section{RESULTADOS E DISCUSSÃO}

Na Tabela 1 encontram-se os resultados obtidos das análises aos 7, 13, 21, 26 e 35 dias após a infestação. No período de 7 a 26 dias após a infestação, apenas a variável massa seca/ninfa (MSN) indicou ser influenciada significativamente pelas variedades. As variedades Desconhecido Branco e Vermelho proporcionaram a menor e a maior massa seca corporal, respectivamente. As demais variedades mantiveram-se numa posição intermediária. Aos 35 dias foram detectadas diferenças significativas somente quanto ao número de ninfas vivas (NNV) e à massa seca (MSN). Na variedade Nenenzinho, o NNV foi maior do que na variedade Arroz Comum. A MSN na variedade Come Cru Vermelho foi maior do que nas variedades Arroz Comum e BR IRGA 409, o que pode significar serem estas menos favoráveis à sobrevivência e desenvolvimento das ninfas, refletindo em menor número de ninfas e massa corporal. Esses resultados estão em conformidade com a situação de que indivíduos com menor peso, criados sobre plantas resistentes, evidenciam uma característica da antibiose, aliada à mortalidade de formas jovens e redução do tamanho (LARA, 1991).

Tabela 1 - Número de ninfas vivas (NNV), massa seca (mg)/ninfa (MSN), superfície corporal/ninfa (SPN) e índice de sobrevivência e desenvolvimento (ISD) de ninfas de Tibraca limbativentris em plantas de dezesseis variedades de arroz avaliadas aos 7, 13, 21, 26 e 35 dias pós-infestação. Santo Antônio de Goiás, GO, 2005.

\begin{tabular}{|c|c|c|c|c|c|c|c|c|}
\hline \multirow[b]{2}{*}{ Variedades } & \multicolumn{4}{|c|}{$7,13,21$ e 26 dias } & \multicolumn{4}{|c|}{35 dias } \\
\hline & NNV & MSN & SPN & ISD & NNV & MSN & SPN & ISD \\
\hline Arroz Misturado & $5,8 \mathrm{a}$ & $77,5 \mathrm{ab}$ & $17,1 \mathrm{a}$ & $3,4 a$ & $4,0 \mathrm{ab}$ & $50,8 \mathrm{ab}$ & $88,7 a$ & $3,9 a$ \\
\hline Agulha & $6,0 \mathrm{a}$ & $76,3 \mathrm{ab}$ & $13,3 a$ & $4,0 \mathrm{a}$ & $4,5 \mathrm{ab}$ & $57,9 \mathrm{ab}$ & $93,7 \mathrm{a}$ & $4,5 \mathrm{a}$ \\
\hline Arroz Comum & $5,5 \mathrm{a}$ & $118,5 \mathrm{ab}$ & $18,4 a$ & $3,8 \mathrm{a}$ & $2,8 b$ & $43,3 b$ & $69,0 \mathrm{a}$ & $2,6 a$ \\
\hline Branco Tardão & $6,3 a$ & $116,8 \mathrm{ab}$ & $21,1 \mathrm{a}$ & $4,0 \mathrm{a}$ & $4,5 \mathrm{ab}$ & $57,3 \mathrm{ab}$ & $89,3 a$ & $4,4 a$ \\
\hline Bico Ganga & $7,5 \mathrm{a}$ & $119,0 \mathrm{ab}$ & $15,6 a$ & $5,3 a$ & $5,8 \mathrm{ab}$ & $51,5 \mathrm{ab}$ & $86,9 a$ & $5,4 a$ \\
\hline Come Cru Vermelho & $5,8 \mathrm{a}$ & $75,5 \mathrm{ab}$ & $16,1 \mathrm{a}$ & $3,9 a$ & $3,8 \mathrm{ab}$ & $79,8 \mathrm{a}$ & $96,0 \mathrm{a}$ & $3,7 \mathrm{a}$ \\
\hline Cutião & $6,0 \mathrm{a}$ & $112,5 \mathrm{ab}$ & $18,9 a$ & $4,0 \mathrm{a}$ & $5,8 \mathrm{ab}$ & $63,0 \mathrm{ab}$ & $96,4 a$ & $5,4 a$ \\
\hline Desconhecido Branco & $4,8 \mathrm{a}$ & $61,5 b$ & $13,5 \mathrm{a}$ & $3,0 \mathrm{a}$ & $3,8 \mathrm{ab}$ & $63,0 \mathrm{ab}$ & $97,6 a$ & $3,5 a$ \\
\hline Gojoba Ligeiro & $6,5 \mathrm{a}$ & $97,3 \mathrm{ab}$ & $18,8 \mathrm{a}$ & $4,3 a$ & $5,3 \mathrm{ab}$ & $55,8 \mathrm{ab}$ & $87,1 \mathrm{a}$ & $5,1 \mathrm{a}$ \\
\hline Marabá Branco & $5,5 \mathrm{a}$ & $75,0 \mathrm{ab}$ & $19,0 \mathrm{a}$ & $3,1 \mathrm{a}$ & $4,0 \mathrm{ab}$ & $52,6 \mathrm{ab}$ & $89,2 a$ & $4,0 \mathrm{a}$ \\
\hline Nenenzinho & $7,3 a$ & $96,3 a b$ & $18,3 a$ & $5,1 \mathrm{a}$ & $6,3 a$ & $52,1 \mathrm{ab}$ & $80,9 a$ & $5,6 a$ \\
\hline Pingo D’água & $7,3 \mathrm{a}$ & $89,0 \mathrm{ab}$ & $14,7 \mathrm{a}$ & $4,9 a$ & $5,8 \mathrm{ab}$ & $64,1 \mathrm{ab}$ & $91,3 a$ & $5,3 a$ \\
\hline Taboca & $5,3 a$ & $93,3 \mathrm{ab}$ & $20,7 \mathrm{a}$ & $3,9 a$ & $4,5 \mathrm{ab}$ & $63,8 \mathrm{ab}$ & $96,5 a$ & $4,1 \mathrm{a}$ \\
\hline Vermelho & $7,3 a$ & $137,8 \mathrm{a}$ & $21,8 \mathrm{a}$ & $5,0 \mathrm{a}$ & $5,8 \mathrm{ab}$ & $59,6 \mathrm{ab}$ & $90,9 a$ & $5,6 a$ \\
\hline Vermelho Agulha & $6,3 a$ & $77,8 \mathrm{ab}$ & $14,4 \mathrm{a}$ & $3,9 a$ & $4,8 \mathrm{ab}$ & $59,0 \mathrm{ab}$ & $91,2 \mathrm{a}$ & $4,7 \mathrm{a}$ \\
\hline BR IRGA 409 & $6,8 \mathrm{a}$ & $98,8 \mathrm{ab}$ & $15,0 \mathrm{a}$ & $4,4 \mathrm{a}$ & $5,3 \mathrm{ab}$ & $38,9 \mathrm{~b}$ & $68,4 a$ & $5,3 a$ \\
\hline Média & 6,2 & 95,2 & 17,3 & 4,1 & 4,8 & 57,0 & 88,3 & 4,6 \\
\hline $\mathrm{CV}$ & 13,3 & 23,9 & 15,4 & 14,8 & 28,3 & 22,8 & 19,2 & 23,3 \\
\hline
\end{tabular}

Nas colunas, médias seguidas de mesmas letras não diferem pelo Teste de Duncan ao nível de $5 \%$ de probabilidade. 
Tabela 2 - Análise conjunta ${ }^{1}$ do número de ninfas (NNV), massa seca (mg)/ninfa (MSN) superfície corporal (SPN), índice de sobrevivência e desenvolvimento (ISD) e dias de vida (DDV) de ninfas de Tibracalimbativentris em plantas de dezesseis variedades de arroz avaliadas aos 7, 13, 21, 26 e 35 dias pós-infestação. Santo Antônio de Goiás, GO, 2005.

\begin{tabular}{|c|c|c|c|c|c|}
\hline Variedades & NNV & MSN & SPN & ISD & DDV \\
\hline Arroz Misturado & $4,9 \mathrm{abc}$ & $34,0 \mathrm{ab}$ & $60,8 \mathrm{a}$ & $3,7 \mathrm{ab}$ & $112,4 \mathrm{ab}$ \\
\hline Agulha & $5,3 a b c$ & $35,6 a b$ & $64,5 \mathrm{a}$ & $4,3 \mathrm{ab}$ & $126,1 \mathrm{ab}$ \\
\hline Arroz Comum & $4,1 c$ & $30,8 \mathrm{ab}$ & $56,0 \mathrm{ab}$ & $3,2 b$ & $96,4 b$ \\
\hline Branco Tardão & $5,4 a b c$ & $39,2 \mathrm{ab}$ & $67,2 \mathrm{a}$ & $4,2 \mathrm{ab}$ & $132,0 \mathrm{ab}$ \\
\hline Bico Ganga & $6,6 a b$ & $33,6 a b$ & $62,9 a$ & $5,4 \mathrm{a}$ & $163,1 \mathrm{a}$ \\
\hline Come Cru Vermelho & $4,8 \mathrm{abc}$ & $47,9 a$ & $67,3 a$ & $3,8 \mathrm{ab}$ & $109,3 \mathrm{ab}$ \\
\hline Cutião & $5,9 a b c$ & $40,9 \mathrm{ab}$ & $69,0 \mathrm{a}$ & $4,7 \mathrm{ab}$ & $152,4 a$ \\
\hline Desconhecido Branco & $4,3 b c$ & $38,2 \mathrm{ab}$ & $63,8 \mathrm{a}$ & $3,3 \mathrm{ab}$ & $103,1 \mathrm{ab}$ \\
\hline Gojoba Ligeiro & $5,9 a b c$ & $37,3 a b$ & $62,7 a$ & $4,7 \mathrm{ab}$ & $142,4 \mathrm{ab}$ \\
\hline Marabá Branco & $4,8 \mathrm{abc}$ & $35,8 \mathrm{ab}$ & $65,8 a$ & $3,6 a b$ & $103,8 \mathrm{ab}$ \\
\hline Nenenzinho & $6,8 \mathrm{a}$ & $35,2 a b$ & $61,7 \mathrm{a}$ & $5,3 a$ & $160,4 a$ \\
\hline Pingo D’água & $6,5 \mathrm{ab}$ & $39,4 \mathrm{ab}$ & $65,6 a$ & $5,1 \mathrm{a}$ & $155,8 \mathrm{a}$ \\
\hline Taboca & $4,9 a b c$ & $42,2 \mathrm{a}$ & $70,6 a$ & $4,0 \mathrm{ab}$ & $124,1 \mathrm{ab}$ \\
\hline Vermelho & $6,5 \mathrm{ab}$ & $40,7 \mathrm{a}$ & $65,9 a$ & $5,3 a$ & $160,0 a$ \\
\hline Vermelho Agulha & $5,5 \mathrm{abc}$ & $36,7 \mathrm{ab}$ & $61,1 \mathrm{a}$ & $4,3 a b$ & $131,1 \mathrm{ab}$ \\
\hline BR IRGA 409 & $6,0 \mathrm{abc}$ & $26,9 b$ & $42,1 b$ & $4,8 \mathrm{ab}$ & $145,4 \mathrm{ab}$ \\
\hline Média & 5,5 & 37,1 & 62,9 & 4,3 & 132,4 \\
\hline $\mathrm{CV}$ & 16,9 & 21,3 & 18,0 & 18,5 & 22,8 \\
\hline
\end{tabular}

${ }^{1}$ Análise conjunta das oito repetições. Nas colunas, médias seguidas de mesmas letras não diferem pelo Teste de Duncan ao nível de $5 \%$ de probabilidade.

Quando as variedades foram analisadas conjuntamente (Tabela 2), todas as variáveis consideradas na avaliação da antibiose das variedades de arroz sobre ninfas de $T$. limbativentris foram significativamente influenciadas. As diferenças entre o menor e o maior número de ninfas vivas (NNV) que era restrito às variedades Arroz Comum e Nenenzinho, respectivamente, expandiram-se. As variedades Arroz Comum e Desconhecido Branco apresentaram o melhor desempenho quando comparadas às variedades Bico Ganga, Nenenzinho, Pingo D' Água e Vermelho que apresentaram maior NNV, porém diferindo entre si.

A massa seca/ninfa (MSN) na variedade Arroz Comum, apesar de não diferir do maior valor obtido na variedade Come Cru Vermelho, foi a mais próxima da menor massa corporal detectada na variedade BR IRGA 409. A superfície corporal (SPN) foi influenciada pela variedade BR IRGA 409, exceto quando comparada com a variedade Arroz Comum. A variedade Arroz Comum apresentou o menor índice de sobrevivência e desenvolvimento (ISD). A variedade Desconhecido Branco, apesar de não diferir das variedades Bico Canga, Nenenzinho e Vermelho, quanto ao ISD, apresentou o valor mais próximo ao do menor índice obtido da variedade Arroz Comum.

$\mathrm{O}$ número de dias de vida das ninfas de $T$. limbativentris (DDV) também foi menor na variedade
Arroz Comum, diferindo do constatado nas variedades Bico Ganga, Cutião, Nenenzinho, Pingo D'Água e Vermelho.

\section{CONCLUSÃO}

Asvariedades Arroz Comume Desconhecido Bran$\mathrm{co}$, principalmente a primeira, evidenciam possuir características que lhes confere determinado grau de resistência do tipo antibiose a ninfas de $T$. limbativentris, devendo ser implementadas pesquisas mais detalhadas sobre este aspecto.

\section{AGRADECIMENTOS}

À Embrapa Arroze Feijão, à UEMA e ao ilustre Dr. Evane Ferreira (in memoriam) que, com compreensão e humildade, contribuíram para a conclusão desta pesquisa.

\section{REFERÊNCIAS}

COSTA, E.C.; LINK, D. Avaliação dos danos de Tibraca limbativentris Stal, 1860 (Hemiptero-Pentatomidae) em arroz irrigado. Anais da Sociedade Entomológica do Brasil, v.21, n.1, p.187-195, 1992. 
FERREIRA, A; LARA, F.M. Tipos de resistência a Alabama argillacea (Huebner, 1818) (Lepidoptera: Noctuidae) envolvidos em genótipos de algodoeiro: II. Antibiose. Bragantia, v.58, n.2, 1999. Disponível em: <http:/ /www.scielo.br>. Acesso em: 22. jul. 2007.

FERREIRA, E.; MARTINS, J.F.S. Insetos prejudiciais ao arroz no Brasil e seu controle. Goiânia: EMBRAPACNPAF, 1984. 67p. (EMBRAPA-CNPAF. Documentos, $11)$.

FERREIRA, E.; MARTINS, J.F.S.; RANGEL, P.H.N.; CUTRIM, V.A. Resistência de arroz ao percevejo do colmo. Pesquisa Agropecuária Brasileira, v.21, n.5, p.565$569,1986$.

FERREIRA, E.; ZIMMERMANN, F. J. D.; SANTOS, A. B.; NEVES, B. P. O percevejo do colmo na cultura do arroz. Goiânia: EMBRAPA-CNPAF, 1997. 43p. (EMBRAPA CNPAF. Documentos, 75).

LARA, F.M. Princípios de resistência de plantas aos insetos. 2 ed. São Paulo: Ícone, 1991. 336p.

SAS. Light user's guide: version 6. Cary, NC, 441p, 1990.

SOUZA, I.S; RODRIGUES, F.J.O. Estudo da resistência varietal ao percevejo Tibraca limbativentris em arroz de sequeiro. In: REUNIÃO NACIONAL DE PESQUISA DE ARROZ, 1990, Goiânia. Resumos. Goiânia: Embrapa Arroz e Feijão, 1990. p.33.

SOUZA, J.R.; FERREIRA, E.; CHAGAS, E.F. Avaliação de variedades de arroz cultivadas no Estado do
Maranhão para antibiose a ninfas de Tibraca limbativentris (Stal, 1860) (Heteroptera: Pentatomidae). In: CONGRESSO BRASILEIRO DA CADEIA PRODUTIVA DE ARROZ, 2.; REUNIÃO NACIONAL DE PESQUISA DE ARROZ/RENAPA, 7., 2006, Brasília. Resumos. Brasília: 2006. p.31-36.

SUINAGA, F.A.A; PICANÇO, M.C.; MOREIRA, M.D.; SEMEÃO, A.A; MAGALHÃES, S.T.V. Resistência por antibiose de Lycopersicon peruvianum á traça do tomateiro. Horticultura Brasileira, v.22, n.2, p.281-285, 2004.

TREMILIOSI, L.M.M. Caracterização de isolados de Bacillus thuringiensis patogênicos à Spodoptera frugiperda (Lepdoptera: Noctuidae). 2006. 61f. Dissertação (Mestrado) - Universidade Estadual Paulista, Faculdade de Ciências Agrárias e Veterinárias, Jaboticabal, 2006.

TRUJILLO, M.R. Contribuição ao conhecimento do dano e biologia de Tibraca limbativentris Stal, 1860 (HemipteroPentatomidae) praga da cultura do arroz. 1970. 63f. Dissertação (Mestrado em Agronomia - Entomologia) Escola Superior de Agricultura Luiz de Queiroz, Universidade de São Paulo, Piracicaba, 1970.

VIANA, P.A.; POTENZA, M.R. Avaliação de antibiose e não-preferência em cultivares de milho selecionados com resistência á lagarta-do-cartucho. Bragantia, v.59, n.1, p.27-33, 2000. Disponível em: <http://www.scielo. br/pdf/brag/v59n1/591 a 5.pdf>. Acesso em: 18. jul. 2007.

Recebido em 10/9/07

Aceito em 20/8/08 